\title{
Experiencia exploratoria de validación de un instrumento sobre nivel de cultura financiera en la generación millennial
}

\author{
SANTOYO-LEDESMA, DiANA S. \\ Universidad Autónoma de Baja California Sur (México) \\ Correo electrónico: dsantoyo@uabcs.mx \\ LUNA-NEMECIO, JOSEMANUEL \\ Centro Universitario CIFE \\ Correo electrónico: josemanuelluna@cife.edu.mx
}

\begin{abstract}
RESUMEN
El presente estudio muestra una experiencia exploratoria de validación de un instrumento de medición del nivel de cultura financiera en la Generación Millenial. El instrumento se elaboró con base en 25 ítems de acuerdo con la Escala de Likert, dirigido a universitarios en el último año de carrera y recién egresados. Por medio de la validez de contenido por 16 jueces expertos y el análisis factorial se logró realizar un ejercicio exploratorio de validez de contenido, permitiendo establecer un análisis de cada uno de los ítems con base en el nivel de cultura financiera. El grado de confianza del instrumento obtenido mediante el Alpha de Cronbach, arrojó un estadístico de (0.681), por lo que se establece que cuenta con un nivel (0.592) de confianza, lo que significa que el contenido del instrumento puede ser empleado para la recogida de datos.
\end{abstract}

Palabras clave: estudio de validez, confianza, cultura financiera, instrumento de evaluación, generación millenial.

Clasificación JEL: A22; A23; G53.

MSC2010: 01-02. 


\title{
Exploratory experience of validation of an instrument on the level of financial literacy in the millennial generation
}

\begin{abstract}
The present study shows an exploratory experience of validation of an instrument for measuring the level of financial culture in the Millennial Generation. The instrument was developed based on 25 items in accordance with the Likert Scale, aimed at university students in the last year of the degree and recent graduates. Through the validity of content by 16 expert judges and the factor analysis, an exploratory exercise of content validity was achieved, allowing an analysis of each of the items to be established based on the level of financial culture. The degree of reliability of the instrument obtained through Cronbach's Alpha, showed a statistic of (0.681), so it is established that it has a level (0.592) of reliability, which means that the content of the instrument can be used for data collection.
\end{abstract}

Keywords: validity study, reliability, financial culture, evaluation instrument, millennial generation.

JEL classification: A22; A23; G53.

MSC2010: 01-02.

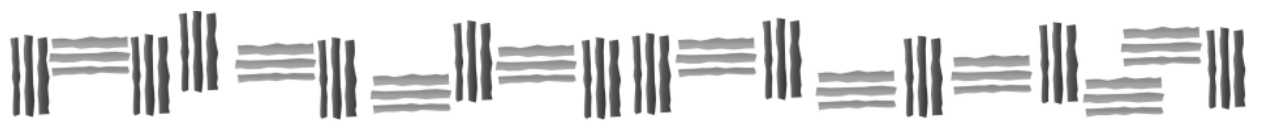




\section{Introducción.}

El interés por ilustrarse en aspectos económicos y financieros ha llevado a varios autores, instituciones y centros de investigación a fijar su mirada en conceptos de educación, inclusión, alfabetización y cultura financiera. De acuerdo a Atkinson \& Messi (2012), el nivel de educación financiera de una persona es medible a partir de sus conocimientos en el contexto de las finanzas, en términos de la realización y aplicación de presupuestos, el control del dinero, la planificación por periodos, ya sea a corto o largo plazo, así como la elección de los productos financieros que le otorgan un mayor beneficio. Según la agencia especializada S\&P Global Ratings (2018) registró a nivel mundial que, sólo un 35\% de los hombres y $30 \%$ de las mujeres poseen conocimiento de educación financiera. Adicionalmente la Asociación Mexicana del Afore (AMAFORE, 2015) indican que la cantidad de mujeres que ahorran es menor comparado con el de los hombres, no obstante, en niveles socioeconómicos más altos no hay diferencias por género.

Roa (2016) en el marco de la Convención Nacional de Afores (2016), enfatizó cuatro puntos que afectan avla educación financiera: a) exceso de confianza: conduce a invertir en activos peligrosos; b) ilusión del conocimiento: poseer la ilusión de saber todo lo necesario, c) conformidad de grupo: tendencia generalizada para hacer lo que hace la mayoría y con base en ello, tomar decisiones con respecto a esa visión y d) disonancia cognitiva: acto de rechazar la información que genera conflicto, se busca información que sustenta lo que se piensa.

Ahora bien, la claridad de los términos tiene un peso importante, como lo indican Amezcua, Arroyo y Espinoza (2014), que mencionan una correspondencia entre la cultura financiera y la educación financiera, es decir que, "a menor nivel de educación financiera corresponde un menor grado de cultura financiera y por consiguiente una disminución en el bienestar social y desarrollo económico" (p. 21). Es por ello que, el foco de estudio son los millennials por ser una generación de amplios retos con respecto a sus predecesores baby boomers, es una generación con mayor incorporación al ámbito laboral y de acuerdo a su perfil sus motivaciones son más ambiciosas (Blancero, Mouriño, \& Padilla, 2018). Sin embargo, también son los menos interesados en dirigir su ahorro, inversiones, gastos, entre otros. Esta generación es concebida por Howe y Strauss (2009), como la nueva generación de consumidores, considerando a los adultos jóvenes nacidos entre 1982 y 2001.

Así mismo, las personas nacidas desde 1980 son nombradas como nativos digitales, destinados a ser el foco de atención por su clara omisión de instruirse, baja claridad de cómo gastar y usar su recurso monetario, desapego profundo con el concepto del ahorro; no lo visualizan como una base de previsión y permanencia ante el futuro, según Auverlot (2011); adicionalmente este comportamiento obedece al esquema actitudinal que enfatiza Llantada (2013) que sostiene que sus actos revelan una baja dosis de tolerancia, demandan una atención de manera inmediata y desean obtener placer urgente para tener todo a su disposición; y como resultado de su educación permisiva están obteniendo una constante frustración en sus campos laborales.

Cabe resaltar que los millenialls también son denominados o etiquetados como multitasking $o$ multitareas, por su facilidad de involucrarse hábilmente con la tecnología, dentro de este ambiente se sienten en confort, aspecto que se denota con los universitarios quienes digitalmente son altamente activos, como menciona Solomon (2013). Según proyecciones de CONAPO (2018), este grupo etario, concebido entre 1980 y el año 2000, pertenece en 34.9\% a la metrópoli en México. El panorama citado por CONAPO (2018) obliga a enfatizar los esfuerzos de investigación y análisis de las causas sobre la cultura financiera y educación financiera, comprender los orígenes que tienen los millenial para dar pie a la falta de conocimiento sobre aspectos económicos y financieros; dado que, según datos obtenidos por la CONDUSEF (2017), el 81\% de los jóvenes de la generación millenial tiene algún tipo de deuda; se registran casos de endeudamiento que están en un proceso de liquidación o con profundo retraso por falta de recursos. El sobreendeudamiento se ha incrementado, obedeciendo a la propensión de los individuos a mejorar su nivel de vida, en búsqueda de compensar sus deseos, adquiriendo productos y servicios que no tienen posibilidades de solventar, éstos jóvenes adultos han logrado incubar una deuda a costa de su tranquilidad económica (Zakaria, Jaafar \& Marican, 2012). 
Así mismo, la Encuesta Nacional de Inclusión Financiera (ENIF, 2015) establece que un 68\% de los adultos de la generación millenial tienen al menos un producto financiero, incrementándose la contratación de servicios financieros para finales de 2015; pero con referencia al ahorro, el 56\% no poseen ningún tipo de cuenta; el 50\% dejó de trabajar y ya no utiliza la tarjeta por la falta de pago de nómina, la banca móvil corresponde al 9.5\%; aunque el 75\% maneja un celular tipo Smartphone, el $50 \%$ no posee una cuenta y por ende no maneja las apps bancarias, a pesar de ser una generación de nativos digitales. Se observó un profundo desconocimiento de las características de los productos y servicios financieros entre la población.

Otro rasgo indicado por Marshall (2015) revela que el principal reto de la educación financiera se vincula con el trabajo profundo de las conductas, habilidades y actitudes que beneficien la mejor toma de decisiones financieras. Es importante añadir que el ENEF (2017) aborda el concepto de educación financiera a través de las acciones que se conciben en conjunto para que los individuos adquieran capacidades que soporten una adecuada gestión y planificación de sus finanzas, con la finalidad de guiarlos a un uso óptimo de los productos y servicios ofrecidos en el mercado en el campo financiero.

Ahora bien, esta investigación pretende adherirse a un perfil científico y para ello deben considerarse los instrumentos de medición, que deben ser fijados a contextos mínimos de validez y confiabilidad. Para los autores Black y Champion (1976) la validez se refiere al significado de la medida como cierta y precisa. Sin embargo, para Baechle y Earle (2007) la validez es el grado en que un ítem de la prueba mide lo pretende medir; es la característica más importante de una prueba. Hay tipologías como la planteada por Maxwell y Stake (2006) que exponen cinco tipos de validez que pueden ser vinculadas a algunas etapas de la investigación: 1) Validez descriptiva: vinculada a la fase inicial de la investigación, se describe lo que fue observado y experimentado. 2) Validez interpretativa: es la certidumbre si los actores pueden confirmar o reconocer los descubrimientos de la investigación en particular. 3) Validez teórica: concerniente con la inmediatez física y mental del fenómeno estudiado. 4) Generalidad: la validez parte por el nivel de la explicación aceptada para perfilarse como generalizable. 5) Validez evaluativa: es la aplicación de un contexto evaluativo, con bases equivalentes en investigación cuantitativa y cualitativa. Ruiz (1998) se refiere a la validez como al grado en que un instrumento realmente mide la variable que pretende medir. Puntualiza que la validez es un concepto del que pueden obtenerse evidencias relacionadas con: el contenido, el criterio y el constructo. Con base en esto, este escrito se concibe como un elemento exploratorio, no pretende la validez total, sino que su propósito es concebir un proceso de carácter exploratorio, que incluya la validez de contenido y la validez de constructo. En cuanto a la confiabilidad, Hernández et al. (2003) señalan: "La confiabilidad de un instrumento se refiere al grado en que su aplicación repetida al mismo sujeto u objeto, produce iguales resultados" (p.243).

Para el caso de estudio, se evaluaron los términos de educación financiera y cultura financiera como conceptos medulares para la medición. Como elementos base, se aplicó una revisión sobre los comportamientos financieros, se indagaron estadísticas, investigaciones, corporaciones que estudian y evalúan a este mercado, además de las instituciones educativas. Una vez realizada esa etapa, se construyó una escala tipo Likert que estuvo compuesta por 25 ítems, se establecieron cinco áreas acerca de su comportamiento financiero como de su nivel de conocimiento, ahorro, inversión, presupuesto, manejo de crédito (tarjetas y préstamos) y vocabulario/términos financieros con cinco opciones de respuesta. Una vez concluido el diseño del instrumento fue transmitido a juicio de un panel de expertos para su evaluación, a fin de revisar los ítems según su pertinencia respecto del constructo de cultura financiera y educación financiera, solicitando observaciones puntuales para obtener congruencia del ítem o en caso necesario eliminar los ítems que no cubrieran el objetivo del estudio.

Una vez culminado el proceso con los expertos se realizaron sólo correcciones de algunos ítems sin necesidad de eliminar alguno de ellos, indicando la importancia de cada ítem para mantener la objetividad y coherencia del instrumento. La siguiente etapa correspondió a su aplicación en una prueba piloto considerando un grupo de 20 personas con perfiles universitarios, con un rango de edad de 18 a 30 años, se contemplaron universidades gubernamentales y personas próximas a egresar o de reciente 
egreso; en definitiva, el instrumento en calidad de pretest y postest. Culminada esta fase, se procedió a elaborar la base de datos con la información obtenida con el propósito de determinar la confiabilidad y validez de constructo del instrumento, mediante los procedimientos estadísticos Coeficiente Alfa de Cronbach, Análisis Factorial, W de Kendall, respectivamente.

Considerando lo anterior, el presente estudio se enfocó en las siguientes metas: 1) medir el nivel de confiabilidad del instrumento pertinente y práctico para evaluar nivel de cultura financiera en la generación millenial, 2) evaluar la validación de contenido del instrumento otorgado por el grupo de expertos; 3) analizar la concordancia del instrumento mediante la aplicación método estadístico de Kappa de Cohen.

\section{Metodología.}

La investigación se desarrolló con base a seis etapas, dando inicio en enero-junio de 2019. Se consideró una muestra de 206 individuos con carrera universitaria concluida o cursándola, en un rango de edad de 18 a 30 años pertenecientes a la generación millenial. Por cada una de las fases fue aplicada una revisión documental, se diseñó un estudio de tipo instrumental de validez de contenido, se empleó la revisión y análisis del Test de Alfabetización Económica para Adultos (TAE-A) (Gempp et al., 2007) utilizado como modelo para generar el diseño del instrumento, dada la naturaleza del tema.

La estructura del estudio se propone bajo una escala de cinco dimensiones. El orden seleccionado fue ahorro, presupuesto, manejo de crédito (tarjetas y préstamos), inversión y términos financieros. La escala consta de 25 ítems, 5 por cada dimensión, en formato de la escala de Likert de 5 puntos; éste fue examinado por un panel de 16 expertos, transitó por un grupo piloto, se analizó la validez bajo el esquema del Alfa de Cronbach como fue utilizado por Materano, Ruíz, Torres y Valera (2009) así como por la W de Kendall. Para finalizar, se aplica un estudio de concordancia utilizando el coeficiente de Kappa de Cohen. Los pasos se muestran en la Figura 1.

\section{Etapa 1. Diseño de instrumento.}

Las dimensiones se desglosaron en cinco fases y por cada una de ella aplica 5 ítems, manteniendo un total de 25 ítems. Las cuestiones se basaron en razonamientos actuales con respecto a finanzas básicas, apegadas a un esquema cotidiano, esto refiere al uso de términos que se previeron que son de dominio en esta generación millenial. Las preguntas se diseñaron bajo criterios de uso frecuente en vida diaria, con la finalidad de obtener un panorama lo más certero posible sobre el dominio de los mismos. De acuerdo a la investigación documental son temas relevantes que los individuos han dejado sin atención. Se completa mediante el siguiente procedimiento un muestreo probabilístico, que permite realizar generalizaciones desde los resultados obtenidos a través la muestra hacia el resto de la población.

\section{Etapa 2. Desarrollo de ítems basado en la escala de Likert.}

Se dispuso el uso de la escala por ser una herramienta de medición como elemento básico en un estudio científico sustentado por Gómez y Amaya (2013), que da pauta para evaluar las actitudes y niveles de conformidad del encuestado. Su uso promueve matizar la opinión de la persona y medir actitudes, como lo indican Méndez y Peña (2007).

\section{Etapa 3. Análisis del instrumento por expertos.}

Esta etapa toma gran importancia de evaluación y validación como lo indican Galicia, Balderrama y Navarro (2017), donde enfatizan la pertinencia de los ítems. La selección de los expertos permite tener mayor certeza y claridad de que la estructura del instrumento goza de objetividad. Esta etapa fue evaluada por 16 expertos concebidos bajo ciertos criterios. 
Figura 1. Desarrollo de etapas.

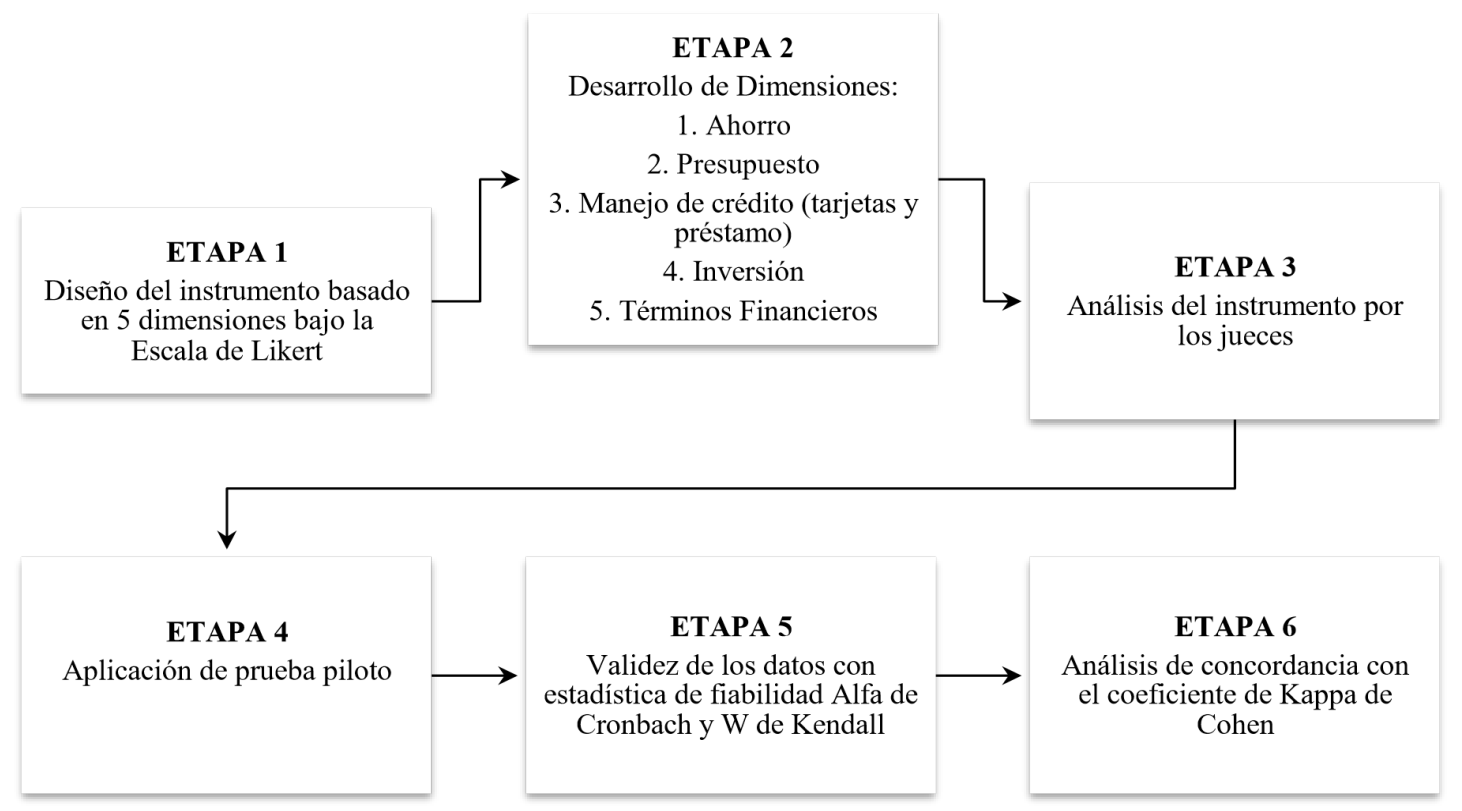

Fuente: Elaboración propia.

\section{Etapa 4. Aplicación de prueba piloto.}

En esta fase de trabajo se conformó un grupo de 20 estudiantes integrados de universidades públicas de la Ciudad de la Paz en Baja California Sur. La aplicación del instrumento se llevó a cabo por medio de Google form; se les convocó de forma personalizada, explicando y puntualizando el objetivo del estudio. Fue importante comulgar con ellos los temas, al inicio del intercambio hubo reacciones de resistencia por el hecho de enfocarlos a finanzas; sin embargo, se les sensibilizó sobre la importancia de sus aportaciones y accedieron de forma positiva a participar. Una vez concluido ese intercambio se les hizo llegar el formulario.

\section{Etapa 5. Validez de los datos con la estadística de fiabilidad del Alfa de Cronbach.}

En este proceso el objetivo era la obtención de una escala de fiabilidad, estimado en un parámetro de $p>0.05$. Tanto para el grupo de expertos como la prueba piloto fueron sometidos a esta fase según Quero (2010).

El Alpha de Cronbach permite realizar determinar la consistencia interna de los ítems y cómo esto se comportan entre ellos.

"El valor mínimo aceptable para el coeficiente alfa de Cronbach es 0.70 ; por debajo de ese valor la consistencia interna de la escala utilizada es baja. Por su parte, el valor máximo esperado es 0.90; por encima de este valor se considera que hay redundancia o duplicación. Varios ítems están midiendo exactamente el mismo elemento de un constructo; por lo tanto, los ítems redundantes deben eliminarse". Usualmente se prefieren valores de alfa entre 0.80 y 0.90. (Oviedo \& Campos 2005, p. 577) 


\section{Etapa 6. Análisis de concordancia con el coeficiente de Kappa de Cohen.}

La medida se basa en la concordancia obtenida en un conjunto de datos. Esto se determina para clarificar la proporción de coincidencias frente a un total de individuos. Así mismo, Cerda \& Villarroel (2008) indican que este proceso pretende eliminar el sesgo dentro de la investigación, para ello se elimina de alguna forma la concordancia obtenida por azar.

La fórmula establecida es la siguiente:

$$
\mathrm{K}=(\mathrm{Co}-\mathrm{Ca}) /(1-\mathrm{Ca})
$$

Si $\mathrm{K}=0$ significa que la concordancia observada coincide con la que ocurriría por puro azar. Los que señalan valores positivos señalan mayor concordancia que la que se esperaría por el puro azar. En caso que el resultado fuera 1, se trataría de una concordancia perfecta. Si K toma un valor negativo, sería igual a discordancia, que solamente en la tabla de $2 \times 2$ podría llegar hasta -1 , lo que señalaría una discordancia total entre las dos clasificaciones o evaluaciones.

Así mismo, hay que calcular también el intervalo de confianza en el que se mueve $\mathrm{K}$, debido a que, en caso que $\mathrm{K}$ tenga valores positivos si el intervalo de confianza es muy amplio, habría que reconsiderar la significación, es decir, si es suficiente para decidir que ambas clasificaciones, observadores, etc. son similares. Ahora bien, según Landis y Kosh (1977) propusieron unos límites para el grado de acuerdo estimado con el resultado del cálculo de Kappa, como se indica en la Tabla 1.

Tabla 1. Interpretación de los valores de Kappa.

\begin{tabular}{cc}
\hline Kappa & Estimación del grado de acuerdo \\
\hline$<0$ & No acuerdo \\
\hline $0.0-0.2$ & Insignificante \\
\hline $0.2-0.4$ & Bajo \\
\hline $0.4-0.6$ & Moderado \\
\hline $0.6-0.8$ & Bueno \\
\hline $0.8-1.0$ & Muy bueno \\
\hline
\end{tabular}

Fuente: Landis \& Koch (1977).

\section{Resultados.}

La obtención de los resultados se fue dando por periodos de tiempo conforme se avanzó en el estudio, desde enero a julio de 2019. Una vez culminadas las etapas, los resultados obtenidos por medio del coeficiente de concordancia de Kendall fue la siguiente: 
Tabla 2. Resultados de la W de Kendall para el instrumento aplicado a expertos.

\begin{tabular}{|l|r|}
\hline \multicolumn{2}{|c|}{$\begin{array}{c}\text { Estadísticos de prueba. Coeficiente de concordancia de } \\
\text { Kendall }\end{array}$} \\
\hline N & 16 \\
\hline W de Kendall & 0.503 \\
\hline Chi-cuadrado & 193.164 \\
\hline gl & 24 \\
\hline Sig. asintótica & 0.000 \\
\hline
\end{tabular}

Fuente: Elaboración propia, basado en el programa SPPS v.24 (2019).

El resultado de 0.503 quedó dentro de los estándares de aceptación: quedando por encima del mínimo con este dato se pudo determinar la existencia de una concordancia entre las variables estudiadas. Para el cálculo de los estadísticos se utilizó el software especializado Statistical Product and Service Solutions (SPSS), versión 24 (George \& Mallery, 2003).

Para la obtención de los valores primero se hizo una tabla en software especializado SPSS, versión 24. Se sacaron las estadísticas descriptivas básicas para después obtener el Alfa de Cronbach y como se puede apreciar en la Tabla 2 se utilizaron los 16 casos para el cálculo del análisis del Alfa de Cronbach, que arrojó un resultado del 0.681 , es decir, al menos el $68.1 \%$ el cual es un porcentaje que dentro de la escala que sugieren George y Mallery (2003) para el análisis del instrumento se considera cuestionable; sin embargo, sigue estando por encima de la escala mínima elegida que es $>.5$ (Tabla 3 ).

Tabla 3. Resultados Alfa de Cronbach para el instrumento aplicado a Jueces.

Estadísticas de fiabilidad
\begin{tabular}{c|c} 
Alfa de Cronbach & $\mathrm{N}^{\mathrm{o}}$. de elementos \\
\hline 0.681 & 26
\end{tabular}

Fuente: Elaboración propia, basado en el programa SPPS v.24 (2019).

Para la prueba piloto a los 20 alumnos de nivel universitario para determinar la validez del contenido, una vez que los expertos hicieron sus observaciones con respecto a los ítems, fueron aplicadas las observaciones; posteriormente se requirió la perspectiva de los jóvenes invitados para aplicar la prueba, que utilizaron las mismas pruebas no paramétricas. Como se puede apreciar en la Tabla 4 de la W de Kendall el resultado ha sido bajo según la escala. 
Tabla 4. Resultados de la W de Kendall acorde al instrumento generado a la prueba piloto.

\begin{tabular}{lr}
\multicolumn{2}{c}{ Estadísticos de prueba } \\
\cline { 2 - 3 } $\mathbf{N}$ & 20 \\
W de Kendall & 0.316 \\
Chi-cuadrado & 151.761 \\
gl & 24 \\
Sig. asintótica & 0.000
\end{tabular}

Fuente: Elaboración propia basado en el programa SPPS v.24 (2019). Coeficiente de concordancia de Kendall.

El resultado no fue con el rango esperado, esto obedece a que la mayor parte de las respuestas que los alumnos pusieron dentro de la escala de Likert fueron ambiguas (ni de acuerdo ni en desacuerdo) ya que usaron la categoría considera media o neutral para responder y debido a esto es posible que la medida de concordancia entre las variables haya resultado tan baja.

Cabe mencionar que, sería admisible sustituir la opción intermedia de la escala de Likert por una que permita al usuario clarificar su respuesta. Esto obedece a que existe una tendencia de las personas encuestadas de optar por la respuesta central cuando desconocen el tema o no pueden identificarse con él. El dato queda dentro de los niveles fiables como se ve en la Tabla 5.

Tabla 5. Resultados Alfa de Cronbach para el instrumento aplicado a la prueba piloto.

\begin{tabular}{|c|c|}
\hline \multicolumn{2}{|c|}{ Estadísticas de fiabilidad } \\
\hline Alfa de Cronbach & $\mathrm{N}^{\circ}$. de elementos \\
\hline $\mathbf{0 . 5 9 2}$ & 26 \\
\hline
\end{tabular}

Fuente: Elaboración propia, basado en el programa SPPS v.24 (2019).

Una vez utilizada esta herramienta de Kappa, nos permite registrar que de los 25 ítems se comportaron en su estimación de grado de resultado como 2 bajo, 8 aceptable, 11 moderado, 3 bueno, 1 muy bueno, por tanto, lo que genera una interpretación de un nivel de acuerdo entre los observadores en la gran mayoría de los ítems, clarificando que las observaciones no estuvieron al azar y su manifestación fue clara. El instrumento con sus resultados se expone de la siguiente forma: 
Tabla 6. Resultados de confiabilidad por ítem a través del grado de concordancia de los observadores.

\begin{tabular}{|c|c|c|c|}
\hline Ítems & Kappa & Ítems & Kappa \\
\hline \multicolumn{2}{|c|}{ Dimensión 1. Ahorro } & \multicolumn{2}{|c|}{ Dimensión 4. Inversión } \\
\hline 1 & 0.40 & 1 & 0.42 \\
\hline 2 & 0.50 & 2 & 0.57 \\
\hline 3 & 0.61 & 3 & 0.40 \\
\hline 4 & 0.44 & 4 & 0.29 \\
\hline 5 & 0.56 & 5 & 0.49 \\
\hline Ítems & Kappa & Ítems & Kappa \\
\hline \multirow{2}{*}{\multicolumn{2}{|c|}{ Dimensión 2. Presupuesto }} & \multirow{2}{*}{\multicolumn{2}{|c|}{$\begin{array}{c}\text { Dimensión } 5 . \\
\text { Vocabulario/términos financieros }\end{array}$}} \\
\hline & & & \\
\hline 1 & 0.45 & 1 & 0.29 \\
\hline 2 & 0.48 & 2 & 0.35 \\
\hline 3 & 0.52 & 3 & 0.55 \\
\hline 4 & 0.40 & 4 & 0.78 \\
\hline 5 & 0.85 & 5 & 0.12 \\
\hline Ítems & Kappa & & \\
\hline
\end{tabular}

Dimensión 3. Manejo de crédito

(tarjetas y préstamos)

\begin{tabular}{ll}
\hline 1 & 0.69 \\
2 & $\mathbf{0 . 1 0}$ \\
3 & 0.41 \\
4 & 0.33 \\
5 & 0.39 \\
\hline
\end{tabular}

Fuente: Elaboración propia, basado en el programa SPPS v.24 (2019). Instrumento de observación aplicados a público universitario a través de dos observadores.

\section{Discusión.}

El proceso realizado en etapas permitió evaluar finalmente el nivel de concordancia con el método del Kappa de Cohen. El desarrollo por etapas generó las bases para lograr el objetivo de esta investigación, respetando el proceso de confiabilidad del instrumento diseñado, evaluación de la validación sometido al grupo de expertos, hasta obtener una concordancia de un $88.7 \%$ como resultado final. El instrumento diseñado logró culminarse con los ajustes propios en el proceso de esta investigación y puede ser utilizado para futuras investigaciones, con la misión de la mejora continua y así mismo de 
perfeccionarse, en la investigación sustentada por Rivera y Bernal (2018) integran ítems como el ahorro, uso de tarjeta de crédito que coinciden con lo sustentado, además consideran el endeudamiento como parte necesaria de evaluación, denotan como resultado que hay un mínimo de manejo en cuanto a la educación financiera. Así mismo, la contribución realizada por los autores Huerta et al. (2018) donde aborda que el género también impacta en la acción del ahorro y la educación financiera enfocado en la generación millenial, aspecto que debe considerarse como punto de validación en las subsecuentes investigaciones.

Cabe señalar que en el estudio se solicitó información adicional para complementar criterios, los resultados se posicionaron de la siguiente forma: que el $92.3 \%$ estudia o estudió en universidades públicas, no se delimitaron las carreras para ser estudiadas, sin embargo, las de mayor abundancia fueron en el área administrativa-económicas, de salud e ingenierías; éste fue el caso de la investigación a cargo de Materano et al. (2009) donde partieron de un estudio de emprendimiento con alumnos de ingeniería agrícola para analizar el nivel educacional que poseen para enfrentar una oportunidad de emprendimiento, es decir, se debe pasar de tener emprendedores a empresarios, a una temprana edad. Como indican Mungaray, Aguilar y Osorio (2017), uno de los principales motivos de iniciar un negocio se asocia a la alternativa de complementar el ingreso familiar y la búsqueda infructuosa de un empleo.

Por otro lado, uno de los datos interesantes de la investigación fue acerca de quién sostiene o sostuvo sus estudios en el lapso de la carrera y el $43.5 \%$ fueron los padres, posteriormente con un $13 \%$ sólo el estudiante lo sostuvo o sostiene y con un $12.2 \%$ sólo la madre contribuyó con la educación. Estos datos se vieron contrarrestados al momento de abordarlos con quien vive actualmente o en su momento de haber sido estudiante, el $61.8 \%$ con sus padres para pasar al $11.1 \%$ a vivir sólo y finalmente un $9.7 \%$ con su propia familia (pareja e hijos). Con estas cifras se deduce que el personaje estudiado de alguna forma ha solventado las dificultades económicas, no por ello significa que domine los conceptos o tenga destreza financiera. Esto lo refiere en su investigación Zapata et al. (2016) donde indica que los individuos poseen una baja cultura financiera, inclinados por el desinterés o por desconocimiento; adicional a esto, tienen desconfianza en el sistema bancario.

Uno de los elementos por puntualizar es la necesidad de fortalecer los programas de estudio para promover una formación en el área financiera. Lusardi y Mitchell (2014) sustentan que las valoraciones de los programas de educación financiera deben poseer claros objetivos para obtener un nivel de eficacia y dar pauta a la mejora de resultados financieros, así como de su comportamiento de consumo.

Hay investigaciones, como la de Bruhn y Love (2014) donde realizaron un estudio en Brasil a 20,000 estudiantes de secundaria; en ella corroboran que si se cuenta con instrucción en educación financiera incrementa la probabilidad de abrir una cuenta bancaria, coadyuvando a tener mayor capacidad para tomar decisiones sobre el dinero. Castillo et al. (2018) abordan la importancia de evaluar los factores internos y externos que fomentan la actividad emprendedora. Otro rasgo de importancia es acerca de la intención emprendedora que sostienen los estudiantes. Lans et al. (2014) mencionan que la enseñanza superior puede aplicarse con mayor importancia para ofrecer las bases necesarias para los futuros emprendedores.

Conviene subrayar que existen otros factores que actúan en el desarrollo de los estudiantes como lo indica Zambrano (2013), que respalda llevar una aproximación para introducir la pedagogía como estrategia educativa para elevar el desempeño educativo. Ahora bien, el plantel educativo se establece como facilitadores del aprendizaje como lo sustenta Cohen, Raudenbush y Ball (2003). Así mismo Hanushek (2008) alude que la calidad del docente y la cantidad de alumnos en el aula son elementos esenciales para el proceso formativo. Adicionalmente Astorquiza (2019) hace mención a que para elevar la calidad de educación se deben repensar los modelos educativos, continuar con fundamentos teóricos y empíricos que permitan en los hacedores de política enfocarse en las peculiaridades del entorno familiar o escolar que permita el ascenso del logro académico del alumnado.

Por otra parte, los estudios de confiabilidad no son abundantes en el campo aquí citado. Generalmente sus pasos llegan, por un lado, hasta la validez del instrumento, o se limitan hasta la 
confiabilidad sin culminar en aspectos de concordancia; donde se aplican con mayor frecuencia es en el área pediátrica y de salud, donde se encuentra ampliamente difundida. Este tipo de diseño de investigación presenta diversas ventajas, acentuando su sencillez logística, facilidad de análisis estadístico y una amplia aplicabilidad en escenarios clínicos, como lo indican Cerda y Villarroel (2008), acentuando que la validez de un estudio puede afectarse severamente si transita por mediciones poco confiables. Así mismo, un principio de error de medición es producto de la variabilidad interobservador, cuya magnitud es posible estimar a través de los llamados estudios de concordancia, que tienen como objetivo estimar hasta qué punto dos observadores coinciden en su medición.

\section{Referencias.}

Asociación Mexicana del Afore (AMAFORE, 2015). Feria de AFORES Ciudad de México. Recuperado de https://bit.ly/2sOn7pU.

Amezcua, E., Arroyo, M., \& Espinosa, F. (2014) Contexto de la Educación Financiera en México. Ciencia Administrativa, 1, 21-30. Recuperado de https://www.uv.mx/iiesca/files/2014/09/03CA201401.pdf.

Atkinson, A., \& Messy, F. (2012) Measuring Financial Literacy: Results of the OECD/International Network on Financial Education. OECD. Insurance and Private Pensions, num 15. París. Publishing 2012.

Auverlot, D., (2011) Le fossé numérique en France, sitio digital Centre d'Analise Stratégique. Rapports et documents. Recuperado de https://bit.ly/2KVJXU0.

Aztorquiza, B. (2019). Efectos de la pedagogía y el compromiso educativo sobre el logro académico de los estudiantes de educación media. Revista de Métodos cuantitativos para la Economía y la Empresa, 28, 47-67.

Baechle, R., \& Earle, W. (2007). Principios del entrenamiento de la fuerza y el acondicionamiento fisico. $2^{a}$, edición. Madrid: Editorial Médica Panamericana.

Blancero, M., Mouriño E., \& Padilla, M. (2018). Latino Millennials The New Diverse Workforce: Challenges and Opportunities. Hispanic Journal of Behavioral Sciences, 40(1), 3-21. DOI: $10.1177 / 0739986317754080$.

Black, A., \& Champion, D. (1976). Method and issues in social research. Nueva York: John Wiley\&Sons.

Bruhn, M., \& Love, I. (2014) The real impacto of improved access to finance: evidence from Mexico. Journal of Finance, forthcoming, 69(3), 1347-1376. DOI: 10.1111 / jofi.12091

Castillo, M., Álvarez, A., Alfaro, M., Sánchez, J., \& Pizarro, I. (2018). Revista de Métodos Cuantitativos para la Economía y la Empresa, 25, 119-129.

Cerda, J., \& Villarroel., L. (2008). Evaluación de la concordancia inter-observador en investigación pediátrica: Coeficiente de Kappa. Revista Chilena de Pediatría 2008, 79(1), 54-58.

Cohen, D., Raudenbush, S., \& Ball, D. (2003). Resources, instruction, and research. Educational Evaluation and Policy Analysis, 25(2), 119-142.

Consejo Nacional de Población, CONAPO (2018). Proyecciones de la población 2010-2050. Recuperdo de https://goo.gl/g7EBYZ 
Comisión Nacional para la Protección y Defensa de los Usuarios de Servicios Financieros, CONDUSEF (2017). Anuario estadístico. Recuperado de https://goo.gl/hcspRf

Estrategia Nacional de Educación Financiera, ENEF (2015). Encuesta Nacional de Inclusión financiera. Recuperado de Estrategia Nacional de Educación Financiera, ENEF (2017).

Estrategia Nacional de Educación Financiera, ENEF (2017). Líneas de acción para fomentar la creación y desarrollo de capacidades financieras en la población mexicana. Recuperado de https://bit.ly/37D6ZGq

Hanushek, E.A. (2008). The Economic benefits of improved teacher quality. In Governance and performance of education systems (pp. 107-135). Stanford University, Stanford, CA, USA: Springer.

Hernández, R., Fernández, C., \& Baptista, P. (2014). Metodología de la investigación (6ª ed.). México D.F.: McGraw-Hill.

Howe, N., \& Strauss, W. (2009). Millenials Rising: The Next Great Generation. New York: Random House.

Huerta, Z., Catache, M., García, M., Martínez, R., González, E., \& Pedroza, L. (2018). Revista Internacional Administración \& Finanzas, 11(2), 59-70.

Galicia, L., Balderrama, J., \& Navarro., R. (2017). Validez de contenido por juicio de expertos: propuesta de una herramienta virtual. Apertura (Guadalajara, Jal.), 9(2), 42-53. DOI: 10.18381/ap.v9n2.993.

Gempp, R., Denegri, N., Caripán, V., Catalán, S., \& Hermosilla, C. (2007). Desarrollo del Test de Alfabetización Económica para Adultos TAE-A-25. Interamerican Journal of Psychology, 41(3), 275-284.

George, D., \& Mallery, P. (2003). SPSS for Windows step by step: A simple guide and reference 11.0 update $\left(4^{\text {th }}\right.$ ed.). Boston: Allyn \& Bacon. Recuperado de: http://www.sciepub.com/reference/65129

Gómez, O., \& Amaya, M. (2013). Instrumentos para elegir y evaluar artículos científicos para la investigación y la práctica basada en evidencia. Aquichan, 13(3), 407-420.

Landis, J., \& Koch, G. (1977). La medición del acuerdo del observador para datos categóricos. Biometrics, 33(1), 159-74. DOI: 10.2307 / 2529310.

Lans, T., Blok, V., \& Wesselink, R. (2014), Learning apart and together: towards an integrated competence framework for sustainable entrepreneurship in higher education. Journal of Cleaner Production, 62(1), 37-47.

Llantada, C. (2013). The article of the future: Strategies for genre stability and change. English for Specific Purpose, 32(4), 221-235.

Lusardi, A., \& Mitchell, O.S. (2014). The Economic Importance of Financial Literacy: Theory and Evidence. Journal of Economic Literature, 52, 1-40. DOI: 10.1257/jel.52.1.5. as.

Marshall, S. (2015). Sobre la cultura del valor material y la cosmografía de la riqueza. Etnografias Contemporáneas, 1(1), 181-226.

Materano, W., Ruíz, L., Torres, C., \& Valera, A. (2009). Una mirada a la formación empresarial para el desarrollo del espíritu emprendedor en las universidades. Visión Gerencial, 2, 279-290. 
Maxwell, L., \& Stake, E. (2006). Research and Statistical Methods in Comunication Sciences and Disorders. Universidad de Michigan: Thomson/Delmar learninf.

Méndez, M. \& Peña, J. (2007). Manual Práctico para el diseño de la Escala de Likert. México: UANL/Trillas.

Mungaray, A., Aguilar, J.G., \& Osorio, G. (2017). Los objetivos económicos de la micro y pequeña empresa en México. Un análisis desde las elasticidades de factores productivos. Revista de Métodos cuantitativos para la Economía y la Empresa, 24, 129-146.

Oviedo, C., \& Campo, Ad. (2005). Aproximación al uso del coeficiente alfa de Cronbach. Revista Colombiana de Psiquiatría, XXXIV(4), 572-580.

Quero, M. (2010). Confiabilidad y coeficiente Alpha de Cronbach. Telos. Recuperado de http://www.redalyc.org/articulo.oa?id=99315569010

Rivera, B., \& Bernal., D. (2018). La importancia de la educación financiera en la toma de decisiones de endeudamiento. Estudio de una sucursal de "Mi Banco" en México. Revista Perspectivas, 41, 117144.

Roa, M. (2016, agosto). Centro de Estudios Monetizados en América Latina (CEMLA). Convención Nacional de Afores (2016), Ciudad de México, México. Recuperado de https://bit.ly/2KgiJGq

Ruiz, C. (1998). Instrumentos de Investigación Educativa. Venezuela: Barquisimeto, CIDEG.

Solomon, M.R. (2013) Comportamiento del Consumidor (7 ${ }^{a}$ Edición). México: Ed. Pearson Prentice Hall.

S\&P (Global Ratings) (2018). Recuperado de https://www.spratings.com/en_US/home

Zakaria, R., Jaafar, N., \& Marican, S. (2012). Financial behavior and financial position: a structural equation modelling approach. Middle-East Journal of Scientific Research, 12(10), 1396-1402.

Zambrano, J.C. (2013). Análisis multinivel del rendimiento escolar en matemáticas para cuarto grado de Educación Básica Primaria en Colombia. Sociedad y Economía, 25, 205-235.

Zapata, A., Cabrera, E., Hernández, J., \& Martínez, J. (2016). Educación financiera entre jóvenes universitarios: Una visión general. Revista Administración y Finanzas, 3(9), 1-8. 